\title{
Tumorßiology
}

\section{A70-C/C8 340}

Activation-induced cytidine deaminase 333

Acute lymphoblastic leukemia 318

Adenovirus 111

Advanced colorectal cancer 212

AID expression 333

Angiogenesis 1, 158

Antiapoptosis 181

Antibody targeting 205

APAF-1 151

Apoptosis 9, 111, 165, 189, 301

- signal-regulating kinase 111

Astrocytic tumors 165

B726P 312

Bcl-2 181

Bladder cancer 9, 52, 57, 151

Blood group antigens 340

Breast 221

- cancer 312, 327

Calcium 173

Cancer 70,123

- diagnostics 57

- genes 57

- prognosis 45

- vaccines 205

Carbohydrates 77

CBP/p300 27

CD147 (EMMPRIN, basigin) 229

CD31 158

CD44v6 253

CD8+ T cells 350

- _ - lymphocytes 70

Chemokines 123

Chemoresistance 181

Chemotherapy 212

Chip 327

c-Jun N-terminal kinase 111

Class II transactivator 27

Claudin 4196

Colon cancer 77

Colorectal cancer 139, 247, 290

- Tumour 93

CXCL12 123

CXCR4 123

Cytokeratin 189

Cytotoxic T lymphocytes 205

DCC 139

Distant metastasis 181

DNA mismatch repair 212, 290

Dynamic heterogeneity 139

E2F-1 111

Early recurrence 247
EGFR 253

Endoglin 1

Endothelial nitric oxide synthase 1

EphA2 165

Epidermal growth factor receptor gene 273

Epigenetics 290

Epitope mapping 100

ERBB2 221

Extracellular matrix metalloproteinase inducer 280

EZH2 151

Flow cytometry 350

Fluorescence in situ hybridisation 221

$\alpha(1,2)$ Fucose 77

Fucosyltransferase 77

Gastric cancer 173, 238, 333

Gene expression 57, 139

- therapy 111

Genotyping 290

Glycosylation 77

Head and neck cancer 273

- $\quad$ - squamous cell carcinoma 253

Helicobacter pylori 333

Hematuria 57

Heparanase 238

Hepatocellular carcinoma xenografts, human 16

Hepatocyte growth factor 36

Hereditary non-polyposis colorectal cancer 290

HIV 70

hMLH1 212

hMSH2 212

HPV DNA chip typing 327

Human chorionic gonadotropin, $\beta$-subunit 52

- leukocyte antigen class I 205

- renal cell carcinoma 181

Hyaluronan 280

Hypoxia-inducible factor 1, 173

Immunoassay 100

Immunohistochemistry 221, 290

Immunophenotype 318

Immunotherapy 70, 205, 238

Inhibitor, apoptosis 132

Interferon- $\alpha_{2 b} 264$

Interferon- $\gamma 27,350$

Ki-67 expression 45
Liver function tests 63

- metastases 36, 247

- surgery 247

Livin 132

M30-Apoptosense ${ }^{\circledR} 189$

Mammaglobin 312

Matrix metalloproteinase 2 280

- metalloproteinases 229

- -, inhibitors 247

Melanoma 264

- inhibitory activity 63

- model 229

Messenger RNA 350

Metastasis 63, 139, 212

Metastatic breast cancer 36

MHC class I 70

Mice 1

Microarray 158

Microsatellite instability 290

Microvessel density 158

Modified recovery 84

Molecular oncology 57

Monoclonal antibodies 100

- antibody 340

mRNA expression 151

MUC1 312

Mutation 290, 301

Mutations 273, 333

Neuroendocrine tumor 196

Neuron-specific enolase 84

Nitric oxide 301

Non-small-cell lung carcinoma 84

NSCLC 340

Nuclear grade 158

Nup8893

Ovarian cancer 280

Overall survival 340

p53 333

- expression 45

Papillomavirus, human 327

PECAM-1 158

Peripheral blood mononuclear cells 350

Polysomy 17221

Potassium chloride 173

Precision profile 84

Prognosis 132

Prognostic factors 247

proGRP 100

Proliferation 165

Promoter methylation 151

PTEN 9
Quantification 253

Quantitative reverse transcriptase polymerase chain reaction 57

Radioimmunoassay 253

Real-time polymerase chain reaction 221

Receptor tyrosine kinase 318

Renal cell carcinoma 132, 158

RhoC 139

Ror1 318

RT-PCR 52, 318

S-100 63

S100A4 27

S-100ß 264

SBEM 312

Scatter factor 36

siRNA 196

Small-cell lung carcinoma 84

Splicing variant 132

Stromal cell-derived factor-1 123

Survivin 9,57

T1N0 breast carcinoma 45

Tetramer 70

Time to progression 340

Tissue microarray 158

Topoisomerase II $\alpha 221$

Transforming growth factor- $\beta$ signaling 77

Transgenic mouse 77

Tumor marker 36, 100

- markers 57

- progression 301

- stroma 229

- suppressor gene 196

- vaccine 238

Tumor-associated antigen 318

Tumorigenesis 1

Tumour aggressiveness 93

Tyrosine kinase domain 273

- $\quad$ inhibitor 273

Ulex europaeus agglutinin-I 77

Urokinase 16

Uveal melanoma 63

Vascular endothelial growth factor 1

Vasostatin 196

Vector-based RNA interference 16

Vinculin 196 